\title{
Caracterización socio-económica de los leprosos atendidos en la unidad de dermatología sanitaria de Maracaibo, Venezuela: un estudio de $\operatorname{casos} 1$
}

\author{
A socioeconomic characterization of leprosy \\ patients at the dermatology clinic in Maracaibo, \\ Venezuela: a case study 1
}

Maria C. Parra 2

\footnotetext{
1 Esta investigación fue auspiciada por el Programa de Pequeñas Becas para Investigaciones en Aspectos Sociales y Económicos de las Enfermedades Tropicales del Laboratorio en Ciencias Sociales, Universidad Central deVenezuela, con apoyo financiero del Programa Especial de Investigación y Entrenamiento en Enfermedades Tropicales UNDP/Banco Mundial/OMS.

2 Universidad del Zulia. Av. 20, no 68-63, Edificio Piacoa, apto 9 , Maracaibo, Venezuela.
}

Abstract This paper presents the soci oeconomic characteristics of leprosy pati ents treated at the Dermatology Clinic in Maracai bo, Venezuela (U.D.S). The characteristics were obtained from a closed questionnaire given to 40 pati ents. Results indicate that this is mainly an adult male population, with a reasonable level of schooling who both work and belong to apparently wellestabl ished, stable family groups; thei $r$ family income level s correspond to a lower or mediumlow social class groups. In addition, the patients are mainly non-disabled, and clinical diagnoses are mostly of the lepromatous and borderline types. Any educational program targeting this group should take these socioeconomic characteristics into account in defining the kind of patients who recei ve treatment at U.D.S.

Key words Leprosy; Socioeconomic Characterization; Family; Social Class; Gender

Resumen El objetivo del trabajo es presentar las características socio-económicas de los leprosos que reciben tratamiento en la Unidad de Dermatología Sanitaria de Maracai bo (UDS), obtenidas a partir de un cuesti onario de preguntas cerradas administrado a una muestra de 40 pacientes (aproximadamente 5\% de la población). Los resultados indican que se trata de una población mayoritariamente masculina, de edad madura, con relativo grado de instrucción, ocupada y perteneci ente a grupos familiares aparentemente constituidos y estables; su ingreso familiar corresponde al de clase baja o clase media baja; además estos pacientes son en su mayoría no incapacitados y la forma clínica desu enfermedad está concentrada en los casos Lepromatosa y Borderline Lepromatosa. Los programas educativos que se planifiquen deben partir de esta caracterización socio-económi ca que aclara quiénes son los enfermos atendi dos en Ia UDS.

Palabras clave Leprosos; Caracterización Socio-Económica; Familia; Clase Social; Género 


\section{Introducción}

Después de más de un siglo de haber sido descubierto por Hansen, el bacilo de la lepra, esta enfermedad sigue ocupando un lugar importante en la temática de la salud y enfermedad de las regiones tropicales y sub-tropicales. Su prevalencia, sobre todo en los países subdesarrollados, se ha convertido en acicate para que los organismos internacionales se propongan la meta, a lograr para el año 2.000 , de su eliminación como problema de salud pública. En Venezuela, aunque se ha evidenciado una disminución exponencial de la enfermedad, desde 1951 (Zuniga \& Castellazi, 1982), todavía encontramos, según cifras del año 92, 8.342 casos registrados y una tasa de prevalencia de 4.04/ 10.000 (U.N., The State of World Population, 1992). De éstos un total de 774 individuos leprosos (aproximadamente $9,2 \%$ ) reciben atención médica en el Servicio de Dermatología Sanitaria del Estado Zulia, constituyéndose esta región del país en la cuarta de mayor prevalencia de lepra, después de Apure y los Estados Andinos. Sin embargo, la gravedad del problema no radica en el número de casos, pocos si consideramos otras enfermedades endémicas, sino en las consecuencias sociales que de ella se derivan, en tanto enfermedades incapacitantes, asociadas a un estigma social cuyas raíces se encuentran en lo más remoto de la historia de la humanidad.

De allí que los organismos encargados de los programas de salud promuevan investigaciones que apuntan a conocer las características socio-económicas de la población enferma, así como sus nociones, creencias y actitudes, elementos constitutivos de las representaciones que de la lepra tienen los leprosos y sus comunidades.

Este es el contexto en el cual, con el auspicio de la Organización Mundial de la Salud (OMS), se planteó la realización de una investigación que permitiera aprehender la representación social que el leproso, bajo control de su dolencia en la Unidad Sanitaria de Maracaibo (Estado Zulia, Venezuela), en el período comprendido entre diciembre de 1993 y febrero 1994, tiene de su enfermedad, así como determinar el papel que juegan la incapacidad física y la inhabilitación social en la mencionada representación.

Ello requirió de una metodología que, en un doble movimiento, permitiera obtener una visión tanto cuantitativa como cualitativa de las nociones, las creencias y otros aspectos subjetivos de la enfermedad, así como de las características personales, familiares, educaciona- les y ocupacionales de los individuos afectados.

En este sentido, el objetivo de este trabajo es dar cuenta de tal caracterización, toda vez que el análisis de los aspectos subjetivos es objeto de otro artículo, de próxima publicación.

\section{Poblacion y método}

Al efecto, de una población de 774 enfermos ambulatorios atendidos en la UDS, se seleccionó una muestra aleatoria simple, ya que no se disponía de información adicional previa, que permitiese conocer la variabilidad interna de los grupos por grados de incapacidad. Se determinó el tamaño de la muestra con un Intervalo de Confianza $=95 \%$ y un Error $=5$ años - consi derando la variable años en el tratamiento - y se distribuyó el total (40 pacientes) de acuerdo con las proporciones del número de enfermos, según su grado de incapacidad. Para la recolección de información se utilizó la técnica de la encuesta, siendo el cuestionario de preguntas cerradas el instrumento seleccionado para obtener los datos de carácter cuantitativo. Así mismo debe aclararse que, dadas las dificultades para contactar a los enfermos en sus hogares o sitios de trabajo o estudio, se escogieron entre los que acudían a recibir su tratamiento en la propia Unidad Dermatológica.

El cuestionario constó de 104 preguntas estructuradas en función de distintos temas de indagación: a) caracterización del entrevistado, b) nociones y creencias sobre la lepra, c) aspectos subjetivos de la enfermedad y d) estigmatización. En este trabajo sólo será analizada la información referida a la caracterización socio-económica del enfermo, substrato en el cual se afincan las dimensiones subjetivas de la enfermedad. Esta caracterización aporta un conocimiento cuantitativo y descriptivo de una muestra de la población enferma, además de proporcionar con precisión la información mínima necesaria acerca del perfil socio-económico de los leprosos que son asistidos en la Unidad Sanitaria de Maracaibo, de tal manera que, como lo sugiere la Organización Panamericana de la Salud (OPS, 1988), todo programa de prevención y tratamiento de la enfermedad parta del conocimiento de quiénes son los enfermos, y no de información estimada.

\section{Resultados y discusión}

Los datos recogidos fueron agrupados de acuerdo a si se trataba de información personal, tales como: edad, sexo, estado civil, grado de ins- 
trucción y ocupación; datos familiares, tales como: número de hijos, composición familiar e ingreso familiar y datos de la enfermedad: forma clínica, grado de incapacidad y antecedentes familiares con lepra.

\section{Datos personales}

Con relación a la edad, se detectó que el $65 \%$ de los enfermos de la muestra se encuentran en las edades comprendidas entre los 30 y los 59 años $y$, de éstos, el mayor porcentaje se ubica entre los 40 y los 49 años. Esta información podría producir varias interpretaciones. En primer lugar, que el promedio de edad delos pacientes indica que el grupo más afectado se encuentra en el período más productivo de la vida, tanto desde el punto de vista físico cuanto intelectual y emocional; en segundo lugar, que, dado el alto promedio de años que estos pacientes han pasado en el servicio (6, 8 años), aunque hayan sido infectados en edades más tempranas, su permanencia en el tratamiento podría significar desarreglos importantes en términos de su rendimiento cotidiano, lo cual, tratándose como ya se dijo de grupos de edades más productivas, podría contribuir a acentuar los elementos socio-culturales asociados a la lepra.

En tercer lugar, se constata lo que en los últimos años se ha venido observando en Venezuela, con relación a la reducción de la participación infantil (menores de 15 años) entre los casos de lepra registrados (Ministerio de Sanidad y Asistencia Social, 1986). Esta información coincide con la que aportan Rojas et al. (1993), en el sentido de que en las regiones de baja prevalencia, la mayor proporción de enfermos se encuentra entre los individuos de mayor edad.

Sin embargo, conviene acotar que tal patrón de distribución etaria no coincide con lo observado en países tales como, Burma, India, Noruega y Filipinas, donde "las tasas de incidencia generalmenteaumentan hasta un máximo entre los 10 y los 20 años de edad y después declinan" (OMS, 1988:20-21); aunque el hecho de la ya mencionada larga permanencia en el tratamiento pudiera, entre otras cosas, explicar tal fenómeno, por lo menos en el caso de estos pacientes.

Al comparar los grupos de edades de acuerdo con el grado de incapacidad, de los casos cuyas edades están comprendidas entre los 10 y los 19 años, la mayoría (80\%) se encuentra en el grado 0 . Ello podría deberse a una detección más temprana de la enfermedad, aunque también pudiera vincularse con la permanencia del contacto, sobre todo si éste se asocia a la presencia de otros enfermos en el entorno familiar. Llama también la atención que, en el grado 2 de incapacidad, la edad de los enfermos se encuentra concentrada en los grupos que van de 40 a 60 y más años. El cálculo de estadísticos, tales como el Coeficiente de Contingencia $(0,45)$, Gamma Condicional $(0,38)$ y Kendall's Tau C $(0,25)$, señala que la asociación entre las variables edad y grado de incapacidad tiende a ser baja.

El $60 \%$ de los enfermos de la muestra es de sexo masculino, lo cual refuerza lo que al respecto se ha encontrado en Latinoamérica, donde la prevalencia de la lepra es de cerca de 1,8/1,0 en hombres y mujeres, respectivamente (Ulrich et al., 1993), al igual que en la mayoría de las regiones del mundo, con excepción de algunas zonas de África (OMS, 1988). Aunque en un estudio reportado en 1990, se detectó, en una región del Brasil, que la tasa de prevalencia por sexo fue de 0,99 hombres por mujeres (Andrade et al., 1990), otras investigaciones reportan para países como Cuba (Rojas et al., 1993) y Venezuela (Ministerio de Sanidad y Asistencia Social, 1986), que en la distribución por sexo hay un ligero predominio de los hombres, señalando, en el caso de Venezuela, una proporción de casos del sexo masculino, expresada en una tasa de 2:1. Sin embargo, en una investigación más reciente, Vivianne de Rojas et al. (1994) señalan, para la ciudad de La Habana, $51 \%$ de enfermos del sexo femenino. Esto podría sugerir que no hay relación estrecha entre sexo y lepra, aunque no ha sido descartado que pueda existir entre género y la mencionada enfermedad.

Tal apreciación pudiera reforzarse si observamos que el número de pacientes del sexo masculino se distribuye entre los distintos grados de incapacidad, mientras que para las pacientes hay una concentración mayor en el grado 0 . Así, el cálculo de algunos estadísticos permite apuntar la posible existencia de cierto grado de asociación entre las variables sexo y grado de incapacidad (Gamma Condicional = 0,62; Tabla 1).

Un porcentaje significativo de los encuestados (50\%) es soltero, sin embargo, 50\% han mantenido o mantienen alguna relación que fue o es, aparentemente estable, toda vez que el $32,5 \%$ son casados o unidos y el $17,5 \%$ son divorciados, separados o viudos. Esta información llama la atención por el hecho de que, aun cuando la mayoría de los enfermos se encuentra en edades en las cuales normalmente se mantiene una relación de pareja, sin embargo sólo cerca de la tercera parte presenta esta ca- 
racterística, lo cual podría estar asociado a las implicaciones socio-culturales de la lepra. Además, si ello es así, coincidiría con lo que otros autores han podido detectar, en el sentido de que el diagnóstico de la enfermedad podría producir desequilibrios emocionales que ocasionen problemas que puedan originar la separación de la pareja (Rojas et al., 1993). Al respecto Ilama la atención, sin embargo, que el porcentaje mayor de quienes han estado unidos al guna vez y ya no lo están corresponde al grado 0 de incapacidad. Sin embargo, tal como los cálculos estadísticos lo señalan, no existe asociación entre el grado de incapacidad de estos enfermos y su estado civil.

En cuanto al grado de instrucción, se encontró que sólo un $15 \%$ es analfabeto, mientras que el $75 \%$ tiene algún grado de instrucción (básica o media), lo cual, aunque podría ser indicativo de una población relativamente instruída, no coincide, pero se aproxima a lo que es la tendencia general de la población del país, la cual presenta un índice de analfabetismo re-

Tabla 1

Sexo de los leprosos, según su grado de incapacidad.

\begin{tabular}{|c|c|c|c|c|}
\hline \multirow[t]{2}{*}{ Sexo } & \multicolumn{3}{|c|}{ Grados de Incapacidad } & \multirow[t]{2}{*}{ Total } \\
\hline & 0 & 1 & 2 & \\
\hline Femenino & 52,0 & 33,3 & 11,1 & 40,0 \\
\hline Masculino & 48,0 & 66,7 & 88,9 & 60,0 \\
\hline Total & 62,5 & 15,0 & 22,5 & 100,0 \\
\hline
\end{tabular}

Gamma Condicional: 0,62264

Tabla 3

Situación laboral de los leprosos después de ser diagnosticada la enfermedad, según su grado de incapacidad.

\begin{tabular}{|c|c|c|c|c|}
\hline \multirow{2}{*}{$\begin{array}{l}\text { Situación } \\
\text { laboral }\end{array}$} & \multicolumn{3}{|c|}{ Grados de incapacidad } & \multirow[t]{2}{*}{ Total } \\
\hline & 0 & 1 & 2 & \\
\hline Trabaja & 16,0 & 0 & 0 & 10,0 \\
\hline Estudia & 64,0 & 50,0 & 88,9 & 67,5 \\
\hline $\begin{array}{l}\text { O ficios del } \\
\text { hogar }\end{array}$ & 8,0 & 16,7 & 0 & 7,5 \\
\hline Desocupado & 12,0 & 33,3 & 0 & 12,5 \\
\hline O tro & 0 & 0 & 11,1 & 2,5 \\
\hline Total & 62,5 & 15,0 & 22,5 & 100,0 \\
\hline
\end{tabular}

Coeficiente de Contingencia: 0,46999 lativamente bajo (OCEI, 1991). Ahora bien, si se compara tal información, de acuerdo con el grado de incapacidad, se encuentra que el $33,3 \%$ de los pacientes grado 2 son analfabetos, lo cual pudiera vincularse al hecho de ser éste grupo el que presenta mayores secuelas de la enfermedad que pudieran haber obstaculizado su incorporación al sistema educativo, además de tratarse del que presenta un promedio mayor de edad. La aplicación de las pruebas estadísticas pertinentes señala que no hay asociación entre el grado de incapacidad y el grado de instrucción para la muestra.

Otro dato importante para definir la caracterización socio-económica de los sujetos era determinar su situación laboral, tanto al inicio de la enfermedad como actualmente, ya que ello permitiría aproximarse a una de las dimensiones sociales de mayor capacidad predictiva, en términos de la estereotipización y el estigma asociados a la lepra y generadores de los temores que los posibles empleadores le tiene a la enfermedad (OMS, 1988). La informa-

Tabla 2

Situación laboral de los leprosos antes de ser diagnosticada la enfermedad, según su grado de incapacidad.

\begin{tabular}{|c|c|c|c|c|}
\hline \multirow{2}{*}{$\begin{array}{l}\text { Situación } \\
\text { labora }\end{array}$} & \multicolumn{3}{|c|}{ Grados de incapacidad } & \multirow[t]{2}{*}{ Total } \\
\hline & 0 & 1 & 2 & \\
\hline Trabaja & 52,0 & 83,3 & 55,6 & 57,5 \\
\hline Estudia & 40,0 & 0 & 11,1 & 27,5 \\
\hline $\begin{array}{l}\text { O ficios del } \\
\text { hogar }\end{array}$ & 4,0 & 16,7 & 0 & 5,0 \\
\hline Desocupado & 4,0 & 0 & 33,3 & 10,0 \\
\hline Total & 62,5 & 15,0 & 22,5 & 100,0 \\
\hline
\end{tabular}

Coeficiente de Contingencia: 0,4997

Tabla 4

Razón del desempleo de los enfermos, según su grado de incapacidad.

\begin{tabular}{lrccr}
\hline Razón & Grados & \multicolumn{4}{c}{ incapacidad } & Total \\
& 0 & 1 & 2 & \\
\hline Ocupado & 88,0 & 83,3 & 88,9 & 87,5 \\
Incapacitado & 4,0 & 16,7 & 11,1 & 7,5 \\
Jubilado & 4,0 & 0 & 0 & 2,5 \\
Falta empleo & 4,0 & 0 & 0 & 2,5 \\
Total & 62,5 & 15,0 & 22,5 & 100,0 \\
\hline
\end{tabular}

Coeficiente de Contingencia: 0,46129 
ción obtenida indica que la mayoría, 57,5\%, se encontraba trabajando al detectarse la enfermedad. Y, aunque el porcentaje de desocupados pasó de ser del 10,0\%, al inicio de la afección, al 12,5\% al momento de recabarse la información, ello puede deberse más a la situación de crisis económica por la cual atraviesa el país, que a su condición de enfermos. No obstante, también podría decirse que el 7,5\% que aduce como razón de su situación de desempleo actual, el estar incapacitado, apunta a reforzar la idea de que el leproso, por su condición de "desacreditado" (Goffman, 1970), es más susceptible de ser objeto de los efectos negativos propios de una economía en recesión. Esto último podría ser complementado con los resultados obtenidos en el cálculo del Coeficiente de Contingencia (0,499; 0,469 y 0,441; Tablas 2, 3 y 4, respectivamente), que indican la existencia de una cierta asociación entre el grado de incapacidad y la situación laboral, antes y después de ser detectada la enfermedad, y la razón para estar desempleado.

Tabla 5

Leprosos que tienen hermanos, según su grado de incapacidad.

\begin{tabular}{|c|c|c|c|c|}
\hline \multirow[t]{2}{*}{ Hermanos } & \multicolumn{3}{|c|}{ Grados de incapacidad } & \multirow[t]{2}{*}{ Total } \\
\hline & 0 & 1 & 2 & \\
\hline $\begin{array}{l}\text { Tienen } \\
\text { hermanos }\end{array}$ & 92,0 & 100,0 & 100,0 & 95,0 \\
\hline $\begin{array}{l}\text { No tienen } \\
\text { hermanos }\end{array}$ & 8,0 & 0 & 0 & 5,0 \\
\hline Total & 62,5 & 15,0 & 22,5 & 100,0 \\
\hline
\end{tabular}

Gamma Condicional: $-1,00000$

Tabla 6

Situación de los enfermos en relación al jefe de familia, según su grado de incapacidad.

\begin{tabular}{lrccr} 
Relación con el & \multicolumn{5}{c}{ Grados de incapacidad } & Total \\
Jefe del Hogar & 0 & 1 & 2 & \\
\hline J efe Hogar & 48,0 & 50,0 & 66,7 & 52,5 \\
Cónyuge & 4,0 & 16,7 & 0 & 5,0 \\
Hijo & 28,0 & 0 & 11,1 & 20,0 \\
Suegro (a) & 4,0 & 0 & 0 & 2,5 \\
Hermano (a) & 8,0 & 33,3 & 0 & 10,0 \\
Otro (a) & 8,0 & 0 & 22,2 & 10,0 \\
& & & & \\
Total & 62,5 & 15,0 & 22,5 & 100,0
\end{tabular}

Coeficiente de Contingencia: 0,47803

\section{Datos familiares}

Conocer las características del grupo familiar al cual pertenece el paciente de lepra resulta prioritario, dado el papel que ello juega en su vida social y la importancia de la familia en cualquier programa de rehabilitación (Gershon \& Srinivasan, 1992).

En este sentido, es interesante destacar que un número considerable de los leprosos de la muestra (50\%) tiene a su madre viva, lo cual, en una sociedad profundamente matriarcal, debe ser tomado en cuenta, en tanto la madre se constituye en el punto de referencia familiar.

Sin embargo, al precisar por grados de incapacidad, esta tendencia se mantiene en los grados 0 y 1 , pero difiere totalmente en el grupo de pacientes grado 2 , toda vez que este es el grupo en el cual la mayor proporción se encuentra en las edades superiores a los 50 años. El cálculo de los estadísticos señala que no hay asociación entre estas dos variables.

La mayoría de los enfermos de la muestra (95\%) tiene hermanos e hijos (70\%), lo cual podría indicar la existencia de vínculos de consanguinidad que se ven reforzados en la medida en que se destaca que el $52,5 \%$ de los encuestados son jefes de familia y el $27,5 \%$ son familiares directos del jefe de familia, ya sea en calidad de cónyuge o de hijo; en otras palabras, el enfermo pertenece a un núcleo familiar establecido, compuesto mayoritariamente por 4 a 6 personas (47,5\%) o 7 a 9 personas (20\%).

Esta tendencia se mantiene al comparar los tres grados de incapacidad; sin embargo, en el caso de la prueba de asociación entre las variables grado de incapacidad y la existencia de hermanos, el estadístico Gamma Condicional señala una asociación negativa (-1; Tabla 5). En términos de la situación del enfermo con relación al jefe del hogar, en los grados 1 y 2 , es menor el porcentaje de los que manifiestan ser cónyuges o hijos. Aquí conviene destacar la importancia de esta información para el desarrollo de políticas de prevención, pero, sobre todo, de tratamiento y rehabilitación, ya que está claro que, al tratarse de ser el enfermo el jefe del hogar, la presencia de la lepra y sus secuelas incapacitantes será más grave para el normal desenvolvimiento de la vida familiar. Esta observación se ve reforzada por el hecho de que el Coeficiente de Contingencia sugiere una leve asociación entre ambas variables (Tabla 6). 
En este orden de ideas, la información sobre el ingreso familiar indica que $40 \%$ de los enfermos de la muestra perciben un ingreso de este tipo, por el orden de los Bolívares (Bs.) 20.000,00 o más, lo cual, si bien no es suficiente para garantizar una condición de vida medianamente holgada, tampoco define una situación de pobreza extrema. Ello podría - entre otros elementos no considerados aquí - sugerir que los enfermos de lepra pertenecen a la clase social media baja. Al respecto conviene señalar que en el grado de incapacidad 2, encontramos los enfermos concentrados $(66,6 \%)$ entre quienes tienen un ingreso que va desde menos de Bs. $8.999,00$ hasta Bs. 14.999,00, mientras que, en los grupos conformados por los enfermos cuyo grado de incapacidad es 1 y 0 , el mayor porcentaje de ellos señala como ingreso familiar, la cantidad de Bs. 20.000,00 a 24.999,00 (grado 1: $50 \%$; grado 0: $32 \%$ ). Este dato es relevante porque, dada la precariedad del ingreso en una economía inflacionaria, se puede concluir que los enfermos con mayor grado de incapacidad añaden a ésto una situación económica que supone condiciones de pobreza, lo cual, además, ratifica lo que parece ser una tendencia todavía no suficientemente demostrada - de

Tabla 7

Ingreso familiar de los enfermos, según su grado de incapacidad.

\begin{tabular}{lcccr}
\hline \multirow{2}{*}{ Bolívares } & \multicolumn{5}{c}{ Grados } & \multicolumn{3}{c}{ incapacidad } & Total \\
& 0 & 1 & 2 & \\
\hline$-8.999,00$ & 8,0 & 0 & 33,3 & 12,5 \\
$9.000,00$ a 14999,00 & 16,0 & 0 & 33,3 & 17,5 \\
$15.000,00$ a 19.999,00 & 16,0 & 33,3 & 0 & 15,0 \\
$20.000,00$ a 24.999,00 & 32,0 & 50,0 & 22,2 & 32,5 \\
$25.000,00$ y más & 12,0 & 0 & 0 & 7,5 \\
No sabe/No responde & 16,0 & 16,7 & 11,1 & 15,0 \\
& 62,5 & 15,0 & 22,5 & 100,0
\end{tabular}

Coeficiente de Contingencia: 0,48254 que existe una estrecha relación entre niveles socio-económicos de la población y lepra. En este sentido, el cálculo de los estadísticos sugiere que hay definida una posible relación (Coeficiente de Contingencia: 0,48; Tabla 7).

\section{Datos sobre la enfermedad}

Según han reportado algunos expertos, se sospecha que la lepra tiene la tendencia a agruparse y a asociarse a factores genéticos que podrían tener "un importante papel en la determinación de la susceptibilidad" (OMS, 1988: 21 ). Al respecto, aunque $75 \%$ de los pacientes encuestados manifestaron no tener antecedentes de presencia de lepra en su familia, $25 \%$, si, lo tienen, lo cual es más relevante si se observa que la mayoría de los antecedentes familiares incluye a padres, hermanos y tíos. Tal información podría corroborar la tendencia ya señalada - al agrupamiento o bien a la debilidad inmunológica genéticamente transmitida, lo cual potencia las posibilidades de contagio. Al respecto, no se detectaron diferencias importantes entre los leprosos de acuerdo con su grado de incapacidad, aunque para ambos

Tabla 8

Antecedente familiar de la enfermedad de los leprosos segun su grado de incapacidad.

\begin{tabular}{lcccc}
\hline \multirow{2}{*}{$\begin{array}{l}\text { Antecedentes } \\
\text { familiares }\end{array}$} & \multicolumn{5}{c}{ Grados de incapacidad } & Total \\
& 0 & 1 & 2 & \\
\hline Si & 36,0 & 33,3 & 11,1 & 30,0 \\
No & 64,0 & 66,7 & 88,9 & 70,0 \\
Total & 62,5 & 15,0 & 22,5 & 100,0 \\
\hline
\end{tabular}

Gamma Condicional: 0,40909

Tabla 9

Parentesco del antecedente con lepra y grado de incapacidad de los enfermos.

\begin{tabular}{lcccr}
\hline Parentesco & \multicolumn{5}{c}{ Grados de incapacidad } & Total \\
& 0 & 1 & 2 & \\
\hline Sin antecedentes & 72,0 & 83,3 & 77,8 & 75,0 \\
Padres-hermanos & 0 & 0 & 11,1 & 2,5 \\
Tios y otros & 0 & 0 & 11,1 & 2,5 \\
Padres-tios & 8,0 & 0 & 0 & 5,0 \\
Padres- & 16,0 & 0 & 0 & 10,0 \\
hermanos-otros & & & & \\
Otros & 4,0 & 16,7 & 0 & 5,0 \\
\hline
\end{tabular}


casos (Gamma Condicional $=0,409$, para los que tienen antecedentes (Tabla 8); Coeficiente de Contingencia $=0,491$ para el parentesco con el antecedente (Tabla 9)) sugieren la definición de una posible relación de estas variables con el grado de incapacidad.

Finalmente, la caracterización de acuerdo con la forma clínica de la enfermedad revela una concentración en Lepromatosa (35\%), Borderline Lepromatosa (30\%), Borderline Borderline (12,5\%); el resto presenta Borderline Tuberculoide (5\%), Indeterminada (7,5\%) y un $10 \%$ del cual no se pudo obtener información al respecto. Con relación al grado de incapacidad, el $62,5 \%$ pertenecen al grado 0 , el $15 \%$ al grado 1 y $22,5 \%$ al grado 2 .

\section{Conclusiones finales}

Las características propias de los grupos de individuos concretos, ubicados en un espacio y un tiempo históricamente determinados, son el substrato sobre el cual se constituyen los elementos subjetivos asociados a la lepra, de allí que sea prioritario conocer de manera sistemática quiénes son los leprosos.
De acuerdo con lo anterior se puede concluir que, si los enfermos bajo tratamiento en la Unidad de Dermatología Sanitaria de Maracaibo mantienen las características de esta muestra, entonces constituyen un grupo conformado mayoritariamente por hombres, en plena edad productiva, trabajadores y con relativo nivel educativo.

Con relación a la enfermedad, se trata de pacientes cuyo promedio de permanencia en el tratamiento es bastante largo (6,8 años), aunque la mayoría de ellos no tiene ninguna incapacidad. Ello puede deberse a condiciones propias del Servicio, que podrían ser evaluadas en futuras investigaciones.

En síntesis, se trata de una población potencialmente susceptible de ser objeto de programas educativos que atiendan no sólo al propio paciente, sino a su familia y a la comunidad.

Puede decirse, además, que los datos obtenidos pudieran confirmar la sospecha, no confirmada, de la asociación entre las condiciones socio-económicas y la predisposición a la enfermedad, de allí que el mejoramiento de estas condiciones pareciera ser uno de los factores a tomar en cuenta con relación al control y a la eliminación de la lepra.

\section{Referencias}

ANDRADE, V.; CHAGASTELLES-SABROZA, P.; CASTRO, A. \& MOTTA, C., 1990. Leprosy spread in urban area. Part I: Epidemiological characteristics of an endemic urban area for leprosy: the county of São Gonçalo, Rio de Janeiro State, Brazil. Hansenologia Internationalis, 15:24-45.

GERSHON, W. \& SRINIVASAN, G., 1992. Communitybased rehabilitation: an evaluation study. Leprosy Review, 63:51-59.

GOFFMAN, E., 1970. Estigma. Buenos Aires: Amorrortu.

MINISTERIO DE SANIDAD Y ASISTENCIA SOCIAL, 1986. Programa de Control de la lepra en Venezuela. VII Congreso Venezolano de Salud Pública. Ponencias, Caracas. (mimeo.)

OCEI (Oficina Central de Estadística e Informática), 1991. Censo de Población y Vivienda. Caracas. OCEI.

OMS (Organización Mundial de la Salud), 1988. Una Guía para el Control dela Lepra. Madrid: Ministerio de Sanidad y Consumo.
OPS (Oficina Panamericana de Salud), 1988. Situación delos programas de control dela lepra en las Amé ricas. OPS. (mimeo.)

ROJAS, V.; ALVISA, R.; HERNANDEZ, O.; BRAVO, R. \& GARCIA, M., 1993. La lepra en ciudad de La Habana, Cuba. In: Las Enfermedades Tropicales en la Sociedad Contemporánea (R. Briceño-León \&J. C. P. Dias, Compiladores), pp. 189-222, Caracas: Fondo Editorial Acta.

ROJAS, V.; HERNANDEZ, O. \& GI L, R., 1994. Algunos factores que inciden en la demora en el diagnóstico de la lepra. Boletín dela Oficina Sanitaria Panamericana, 116:307-312.

UNITED NATIONS, 1992. The State of World Population. New York: United Nations.

ULRICH, M.; ZULUETA, A. M.; CACERES-PITTMAR, G.; SAMPSON, C.; RINARDI, M. E.; RADA, E. \& ARANZAZU, N., 1993. Leprosy in women: characteristics and repercussions. Social Scienceand Me dicine, 37:445-456.

ZUNIGA, M. \& CASTELLAZZI, Z., 1982. 30 Años dela Endemia de Lepra en Venezuela. Caracas: CEPIALET. 